\title{
Photoproduction of $\Theta^{+}$and other pentaquark states
}

\author{
Frank E. Close ${ }^{1 *}$ and Qiang Zhao ${ }^{2 \dagger}$ \\ 1) Department of Theoretical Physics, University of Oxford, \\ Keble Rd., Oxford, OX1 3NP, United Kingdom and \\ 2) Department of Physics, University of Surrey, Guildford, GU2 7XH, United Kingdom
}

(Dated: November 13, 2018)

\begin{abstract}
We present constraints on the relative photoproduction cross sections of positive parity pentaquark states, $\Sigma_{5}, \Lambda_{5}$, and $N_{5}$, based on a minimum phenomenology gained in $\gamma N \rightarrow K \Theta^{+}$and their baryon-meson couplings as in the work of Close and Dudek. The possibility of anomalous signals in $\gamma p \rightarrow K_{S}^{0}\left(\Theta^{+} ; \Sigma_{d}^{+}\right)$is discussed. We emphasise the importance of comparing $\gamma N \rightarrow K \Theta^{+}$with "conventional" states such as $\gamma N \rightarrow K \Sigma(1660)$.
\end{abstract}

PACS numbers: 13.40.-f, 13.88.+e, 13.75.Jz

Even though several photoproduction experiments report signals for the existence of the pentaquark $\Theta^{+}(1540)$ with $\sim 4-5 \sigma$ [1, 2, 3, [4, [5], and narrow baryon states decaying into $p K_{S}^{0}$ are also reported in other reactions [6, 7, , 8, 9], the existence of the $\Theta^{+}(1540)$ is far from proved 10, 11, 12]. A definitive result from a single experiment is still awaited. Meanwhile there is no clear signal of any other potential light pentaquark, save possibly the $\Xi^{--}$states [13], though here again there are criticisms [14]. In this Letter we propose a consistency check for the $\Theta^{+}$and related light pentaquark states by focussing on the relative magnitudes of their photoproduction cross sections.

Pentaquark models, designed to place the $\Theta^{+}\left(q^{4} \bar{q}\right)$ in a $\overline{\mathbf{1 0}}$ of flavour, require the $(q q q q)$ tetraquark to be in $\overline{\mathbf{6}}_{F}$. When combined with the $\overline{\mathbf{3}}_{F}$ of the $\bar{q}$ this implies pentaquark systems in both $\overline{\mathbf{1 0}} \oplus \mathbf{8}$. The narrow widths are then assumed to arise from color-flavor-spin mismatch prior to falling apart to form the octet meson and baryon [15, 16, 17].

Close and Dudek proposed a scheme to construct the flavor wavefunctions of pentaquark states 12, 16]. This leads immediately to the relative strengths of all possible channels to which a pentaquark state can couple. We show here that some of these place severe constraints on the relative production rates for $\gamma N \rightarrow K \Theta^{+} ; K \Sigma_{5} ; K \Lambda_{5}$; and $\pi N_{5}$. We suggest that dedicated experimental searches may either reveal further pentaquarks or alternatively highlight inconsistencies that would call into question the interpretation of the narrow structure named $\Theta^{+}$in $\gamma N \rightarrow K^{\prime \prime} \Theta^{+}$" as a pentaquark.

Several phenomenological studies of $\gamma N \rightarrow K \Theta^{+}$have been proposed in the literature 18, 19, 20, 21, 22, 23, 24, 25, 26]. Although these give quite different predictions in detail due to their varied underlying assumptions, they share certain essential common features that enable robust constraints to be placed on the relative production rates for various pentaquarks. If the $\Theta^{+}$should continue to be claimed in photoproduction data, then these partner states should also appear with similar rates. Failure to observe them would require significant re-evaluation of the dynamics of these hypothesised pentaquarks.

In this work, we will quantify the relative cross sections for photoproduction of $\Theta, \Sigma_{5}, \Lambda_{5}$, and $N_{5}$.

The pentaquark wavefunctions in Ref. [16] are written in a form that exposes their overlaps with the octet mesonbaryon configurations to which the pentaquark state will couple. The explicit decomposition for $\Sigma_{5}, \Lambda_{5}$, and $N_{5}$ is given in Table 1 A tensor formalism that reproduces a subset of these results (specifically the decays $\mathbf{8}_{5} \rightarrow \mathbf{8}_{3} \otimes \mathbf{8}_{3}$ ) has been given in Ref. [27]. As shown in Ref. [16], some selection rules emerge for their decay branching ratios that may be used to help identify the constitution of the states. Future experiments should be able to examine such predictions and provide tests of the underlying dynamics. One advantage of the framework of Ref. 16] is that it provides a connection between the pentaquark $\Theta^{+}$and other pentaquark states such as $\Sigma_{5}$ and $\Lambda_{5}$. Therefore, information available for the $\Theta^{+}$(even though still limited) provides a guide for the production of other pentaquark states.

To proceed, we will first summarize the phenomenology for the $\Theta^{+}$photoproduction in $\gamma n \rightarrow \Theta^{+} K^{-}$and $\gamma p \rightarrow$ $\Theta^{+} \bar{K}^{0}$. Then we abstract the general features and evaluate their implications for $\gamma N \rightarrow K\left(\Sigma_{5} ; \Lambda_{5}\right)$, and $\pi N_{5}$.

For a positive parity $\Theta^{+}\left(1 / 2^{+}\right)$, a pseudovector effective Lagrangian is introduced for the $\Theta N K$ coupling [23]:

$$
\mathcal{L}_{\text {eff }}=g_{\Theta N K} \bar{\Theta} \gamma_{\mu} \gamma_{5} \partial^{\mu} K N+\text { h.c., }
$$

where $N$ denotes the initial nucleon field, while $\bar{\Theta}$ and $K$ denote the final state $\Theta^{+}$and kaon. We adopt $g_{\Theta N K}=1$

\footnotetext{
* e-mail: F.Close1@physics.ox.ac.uk

$\dagger$ e-mail: Qiang.Zhao@surrey.ac.uk
} 
corresponding to total width $\Gamma_{\Theta^{+} \rightarrow K N} \sim 1 \mathrm{MeV}$ in the calculations. This choice is simply to set the scale; in all rates we will show how they rescale with a given total width $\Gamma_{T}$.

The general assumption subsumed in Refs. 18, 19, 20, 21, 22, 23, 24, 25, 26] is that for $\gamma n \rightarrow K^{-} \Theta^{+}$, four transition amplitudes labelled by the Mandelstam variables contribute in the Born approximation limit. These are shown by diagrams in Fig. 1] the contact term, $t$-channel kaon exchange, $s$-channel nucleon exchange and $u$-channel $\Theta^{+}$exchange. The differing output of the calculations in Refs. [18, 19, 20, 21, 22, 23, 24, 25, 26] for magnitudes and energy dependences are due to their various assumptions on the magnitudes of, e.g., $g_{\Theta N K}, g_{\Theta N K^{*}}$, the role of the contact term and importance of $s: u$ channels. However, some qualitative features can be immediately understood, which are sufficient for the generalisations in this present paper.

For $\gamma p \rightarrow \bar{K}^{0} \Theta^{+}$, due to the absence of the contact and $t$-channel neutral-kaon exchange, the Born terms will only consist of the $s$-channel nucleon exchange and $u$-channel $\Theta^{+}$exchange. This feature leads to some model-independent predictions for the Born contributions. In particular, due to the dominance of the contact term in the Born limit, cross sections for $\gamma n \rightarrow K^{-} \Theta^{+}$will be significantly larger than those for $\gamma p \rightarrow \bar{K}^{0} \Theta^{+}$. The $t$-channel transition is also important over a wide energy region.

In $K^{0}\left(\bar{K}^{0}\right)$ production, the absence of the contact term and $t$-channel kaon exchange requires the possibility of $K^{*}$ exchange as essential. This is generally assumed. Therefore, we also include the $K^{*}$ exchange in this model as the leading contribution in association with the Born terms. The $K^{*} N \Theta$ interaction is given by

$$
\mathcal{L}_{\Theta N K^{*}}=g_{\Theta N K^{*}} \bar{\Theta}\left(\gamma_{\mu}+\frac{\kappa_{\theta}^{*}}{2 M_{\Theta}} \sigma_{\mu \nu} \partial^{\nu}\right) V^{\mu} N+\text { h.c. }
$$

where $g_{\Theta N K^{*}}$ and $\kappa_{A}^{*}$ denote the vector and "anomalous moment" couplings, respectively. The "fall-apart" decay dynamics of Refs. [15, 16, 17] implicitly constrains these to be $g_{\Theta N K^{*}}^{2}=3 g_{\Theta N K}^{2}$ and $\left|\kappa_{\theta}^{*}\right|=0$. Note that this will enhance the strength of the $K^{*}$ exchange since most of the previous literature $19,20,21,22$, 25, 26] assume $\left|g_{\Theta N K^{*}}\right| /\left|g_{\Theta N K}\right| \leq 1$. The predicted cross sections also differ from Refs. 23, 24], where $\left|\kappa_{\theta}^{*}\right|=\left|\kappa_{\rho}\right|=3.7$ was adopted, based on an assumed similarity with $\rho N N$. In this work, we adopt $\left|\kappa_{\theta}^{*}\right|=0$, which is more reasonable for the physics of $(q q q q) \bar{q} \rightarrow N K / N K^{*}$ due to the fall-apart dynamics.

The effective Lagrangian for the $K^{*} K \gamma$ vertex is given by

$$
\mathcal{L}_{K^{*} K \gamma}=\frac{i e_{0} g_{K^{*} K \gamma}}{M_{K}} \epsilon_{\alpha \beta \gamma \delta} \partial^{\alpha} A^{\beta} \partial^{\gamma} V^{\delta} K+\text { h.c. },
$$

where $V^{\delta}$ denotes the $K^{*}$ field. For the M1 transition in the quark model one expects that $g_{K^{* \pm} K^{ \pm} \gamma}<g_{K^{* 0} K^{0} \gamma}$, the precise ratio being sensitively dependent on the ratio $m_{s} / m_{d}$. Data agree with this; the $\Gamma_{K^{* \pm} \rightarrow K^{ \pm} \gamma}=50 \mathrm{keV}$ implying $g_{K^{* \pm} K^{ \pm} \gamma}=0.744$, while $g_{K^{* 0} K^{0} \gamma}=1.13$ is determined by $\Gamma_{K^{* 0} \rightarrow K^{0} \gamma}=117 \mathrm{keV}[28]$.

Therefore, within the basic assumption that the meson-baryon- $B_{5}$ couplings (where $B_{5}$ denotes a generic pentaquark baryon) are determined by "fall-apart" topologies, we have constrained the relative photoproduction cross sections for the various $B_{5}$ pentaquark states. The most obvious deficiency in the models is the neglect of Reggeisation for the $K$-exchange. Thus, while the model may be reliable near threshold, the energy dependence for $E_{\gamma}>3 \mathrm{GeV}$ will be increasingly unreliable. However, it is not essentially important for the purpose of this work. Here, our interest is to make a connection between $\Theta^{+}$and other photoproduced pentaquark states based on the available information, in particular, the relative production rates between the $\Theta^{+}$and other pentaquark states. Such relative rates, which are essentially controlled by the "fall-apart" mechanism, should be less dependent on the values of cross sections predicted by the phenomenology. Therefore, they will provide a guide for the experimental search for their signals.

Taking the above ingredients as input, we classify production of pentaquark states $N_{5}, \Sigma_{5}$, and $\Lambda_{5}$ into four classes:

i) charged meson production from neutron target:

$$
\left\{\begin{array}{l}
\gamma n \rightarrow K^{-} \Theta^{+} \\
\gamma n \rightarrow K^{+} \Sigma_{5}^{-} \\
\gamma n \rightarrow \pi^{-} p_{5}
\end{array}\right.
$$

ii) charged meson production from proton target:

$$
\left\{\begin{array}{l}
\gamma p \rightarrow K^{+} \Lambda_{5} \\
\gamma p \rightarrow K^{+} \Sigma_{5}^{0} \\
\gamma p \rightarrow \pi^{+} n_{5}
\end{array}\right.
$$


iii) neutral meson production from neutron target:

$$
\left\{\begin{array}{l}
\gamma n \rightarrow K^{0} \Lambda_{5} \\
\gamma n \rightarrow K^{0} \Sigma_{5}^{0} \\
\gamma n \rightarrow \pi^{0} n_{5} \\
\gamma n \rightarrow \eta_{1} n_{5}(\mathbf{8}) \\
\gamma n \rightarrow \eta_{8} n_{5}(\overline{\mathbf{1 0}})
\end{array}\right.
$$

iv) neutral meson production from proton target:

$$
\left\{\begin{array}{c}
\gamma p \rightarrow \bar{K}^{0} \Theta^{+} \\
\gamma p \rightarrow K^{0} \Sigma_{5}^{+} \\
\gamma p \rightarrow \pi^{0} p_{5} \\
\gamma p \rightarrow \eta_{1} p_{5}(\mathbf{8}) \\
\gamma p \rightarrow \eta_{8} p_{5}(\mathbf{\mathbf { 1 0 }})
\end{array}\right.
$$

where $\eta_{1} \equiv-(u \bar{u}+d \bar{d}+s \bar{s}) / \sqrt{3}$ and $\eta_{8} \equiv(2 s \bar{s}-u \bar{u}-d \bar{d}) / \sqrt{6}$.

The following features can be abstracted for the photoproduction of pentaquarks:

i) In the charged meson production reactions, i.e. Eqs. (4) and (5), the cross section near threshold will be dominated by the contact and $t$-channel charged-meson exchanges in the Born approximation [23].

ii) In the neutral meson production reactions, i.e. Eqs. (6) and (7), the contact and $t$-channel meson exchanges will vanish since they are proportional to the outgoing meson charge. Thus, only the $s$ - and $u$-channel baryon-pole exchanges will contribute to the Born terms.

iii) For the $s$-channel transitions, the difference of the electromagnetic (EM) couplings arises from different targets, i.e. proton or neutron.

iv) For the $u$-channel transitions, the difference of the EM couplings arises from the EM form factors of the pentaquark states, theoretical estimates for some of which have been made in Refs. 23, 29, 30, 31, 32, 33]. In photoproduction reactions, since the $u$-channel transitions are generally suppressed due to the restricted kinematics, the cross section is not a sensitive observable to the pentaquark magnetic moments. The $u$-channel contributions to the total cross section is thus negligible, though they can produce sizeable effects in polarization observables [23, 24].

For $B_{5}=\Theta^{+}, \Sigma_{5}, \Lambda_{5}$, the $s$ and $t$-channel amplitudes are proportional to $g_{B_{5} N K}$. Therefore, to the extent that the $u$-channel is negligibly small, the results of Ref. [16] imply that the relative photoproduction cross sections for $\gamma N \rightarrow K B_{5}$ scale in proportion to $g_{B_{5} N K}^{2}$ and are correlated by effective Clebsch-Gordan coefficients apart from kinematic factors near threshold arising from mass dependences.

v) The $t$-channel vector-meson $\left(K^{*}, \rho\right)$ exchange can contribute to most of those reactions. By comparing the charged and neutral meson production processes, useful information about the vector-meson-pentaquark couplings can be gained.

The absence of Reggeisation means that the cross sections become unreliable at high energy. Thus the absolute cross sections in Figs. 2] 3 and 4 are only guides to show how existing models can be reproduced and generalised. Our principal results will be the ratios of production rates in Figs. [5 Fig. 6] and Fig. 7

Concerning the strong couplings, we take the experimental results for the $\Theta^{+}$as a guide. Since $\Theta^{+}$can only strongly decay into $p K^{0}$ or $n K^{+}$, the coupling constant $g_{\Theta N K}$ can be estimated by $g_{\Theta N K} \equiv g_{A} M_{n} / f_{\theta}$, with

$$
\frac{f_{\theta}}{g_{A}}=\left(1-\frac{p_{0}}{E_{n}+M_{n}}\right)\left[\frac{|\mathbf{p}|^{3}\left(E_{n}+M_{n}\right)}{4 \pi M_{\Theta} \Gamma_{\Theta^{+} \rightarrow K^{+} n}}\right]^{1 / 2},
$$

where $\mathbf{p}$ and $p_{0}$ are the momentum and energy of the kaon in the $\Theta^{+}$rest frame, and $M_{n}$ and $E_{n}$ are the mass and energy of the nucleon, respectively.

Within the fall-apart dynamics, $g_{0} \equiv g_{\Theta N K}$ is the coupling strength setting the scale for the strange-quark-associated pentaquark production and decay amplitudes. Thus, if there were no symmetry breaking in the masses, all pentaquark states considered here would have identical total widths. As $g_{B_{5} N K^{*}}^{2}=3 g_{B_{5} N K}^{2}$, the overall ratios of cross sections survive even after the inclusion of $K^{*}$ exchange contributions.

The partial width of decay channel $i$ is

$$
\Gamma_{i}=g_{i}^{2} \frac{|\mathbf{p}|^{3}\left(E_{f}+M_{f}\right)}{4 \pi M_{n}^{2} M_{i}}\left(1-\frac{p_{0}}{E_{f}+M_{f}}\right)^{2},
$$

where $\mathbf{p}$ and $p_{0}$ are momentum and energy of the final state kaon in the pentaquark rest frame; $E_{f}$ and $M_{f}$ are the energy and mass of the final state baryon; $M_{i}$ is the pentaquark mass and $g_{i}$ is given by $g_{0}$ multiplied by the relevant 
Clebsch-Gordan coefficient. For instance, from Ref. 16]

$$
\Theta^{+}(\overline{\mathbf{1 0}}) \rightarrow \frac{1}{\sqrt{2}}\left\{p K^{0}-n K^{+}\right\}
$$

which leads to

$$
\Gamma_{T}\left(\Theta^{+}\right)=\Gamma_{\Theta^{+} \rightarrow p K^{0}}+\Gamma_{\Theta^{+} \rightarrow=n K^{+}} \sim 1 \mathrm{MeV} .
$$

In Tables 【 and 【II the partial and isospin averaged total widths are given, which are based on Ref. [16], but modified by the phase space factors.

In the fall-apart dynamics all pentaquarks would have the same total widths in the absence of flavour symmetry breaking, which is illustrated by Table $\llbracket$ For both $\Sigma_{5}(\overline{\mathbf{1 0}})$ and $\Sigma_{5}\left(\mathbf{8}_{5}\right), M_{\Sigma_{5}}=1.7 \mathrm{GeV}$ is adopted before mixing.

For the production of $\Sigma_{5}^{0}$ the total width for the $\overline{\mathbf{1 0}}$ is about a factor of 2 bigger than that for the $\mathbf{8}_{5}$. The reason is that for channels, $\Xi^{0} K^{0}$ and $\Xi^{-} K^{+}$, the decay Clebsch-Gordans favor the $\mathbf{8}_{5}$ relative to the $\overline{\mathbf{1 0}}$, but due to the higher threshold of these two channels, the decay widths are strongly suppressed. This cuts into the width of the "favoured" $\mathbf{8}_{5}$ more than the $\overline{\mathbf{1 0}}$, thereby leading to the relatively larger width for the $\overline{\mathbf{1 0}}$. The result is that after phase space is taken into account, the total width of the $\Sigma_{5}(\overline{\mathbf{1 0}})$ is about a factor of two larger than that of the $\Sigma_{5}(\mathbf{8})$ and a factor of three larger than the $\Theta^{+}$.

The mass of the $\Sigma_{5}$ could be an important issue in the search for its signals. In Ref. [17] mixing between the $\mathbf{8}_{5}$ and $\overline{\mathbf{1 0}}$ was assumed to be extreme such that the physical mass eignestates are the light and heavy states with "hidden $d \bar{d}$ " and "hidden $s \bar{s}$ ", respectively. We shall label these $\Sigma_{d}$ and $\Sigma_{s}$. If this is realised in practice there is the possibility that the $\Sigma_{d}$ has a mass similar to that of the $\Theta^{+}$[17]. This could give interesting signals in $\gamma p \rightarrow K_{S}^{0}\left(\Theta^{+} ; \Sigma_{d}^{+}\right)$arising from the primitive reactions $\gamma p \rightarrow \bar{K}^{0} \Theta^{+}$and $\gamma p \rightarrow K^{0} \Sigma_{5}^{+}$. In the case of degeneracy the rate of $\Sigma_{5}^{+}$production could be the same as $\Theta^{+}$production in $\gamma p \rightarrow \bar{K}^{0} \Theta^{+}$due to the interference between these two representations. In general, for similar masses the $\Sigma_{d}$ production could be driven by rescattering $\gamma p \rightarrow \bar{K}^{0} \Theta^{+} \rightarrow K^{0} \Sigma_{5}^{+}$. Such an ambiguity does not exist in the neutron target reaction, where the production of $\Sigma_{5}^{+}$is forbidden. However, if the $\Sigma_{5}$ states are located significantly above the $\Theta^{+}$, and/or rescattering is not important, as we shall show below, a kinematic suppression can lead to a rather small rate for the production of $\Sigma_{5}$.

The production of $\Lambda_{5}(\mathbf{8})$ is also crucially dependent on its mass. In this work, $M_{\Lambda_{5}}=1.65 \mathrm{GeV}$ is adopted. The difficulty of isolating $\Lambda_{5}$ in either $\gamma p \rightarrow K^{+} \Lambda_{5}$ or $\gamma n \rightarrow K^{0} \Lambda_{5}$ is that the conventional octet states $\Lambda_{3}$ can be also produced in association with $K^{+}$or $K^{0}$. Indeed, a $P_{01}$ resonance $\Lambda(1600)$ with positive parity and a width about $150 \mathrm{MeV}$ is known to exist in this energy region and as such may mix with, and hide, the primitive pentaquark state. However, in such a case the photoproduction cross section for the $\Lambda(1600)$ would be at least as big as the pentaquark case presented here since it could then be produced through its pentaquark component as well as "conventionally". Similar remarks apply to $\Sigma^{*}$ states produced via any $\Sigma_{5}$ component.

To proceed, we will analyze reactions of Eqs. (40 5 6] (7) based on our phenomenology for $\gamma p \rightarrow \bar{K}^{0} \Theta^{+}$and $\gamma n \rightarrow K^{-} \Theta^{+}$. As before we follow the fall-apart mechanism, where $g_{\Sigma N K^{*}}^{2}=3 g_{\Sigma N K}^{2}$, and $g_{\Lambda N K^{*}}^{2}=3 g_{\Lambda N K}^{2}$, as a basic condition for the strange-quark-associated reactions.

In Fig. 2 total cross sections for $\Theta^{+}$with $\Gamma_{T} \sim 1 \mathrm{MeV}$ are presented. For other values of the total width, the estimates can be rescaled by $\sigma \rightarrow \sigma \times \Gamma_{T} /(1 \mathrm{MeV})$. As we repeatedly stress: the absence of Reggeisation means that the energy dependence of the cross section in this and subsequent absolute cross sections is unreliable for $E_{\gamma}>3$ $\mathrm{GeV}$.

In the Born limit, the role played by the contact and $t$-channel kaon exchange for the neutron target (i.e. $K^{-}$ production) is highlighted by the significant difference between the two solid curves. Due to $g_{\Theta N K^{*}}^{2}=3 g_{\Theta N K}^{2}[15$, 16], the contributions of the $K^{*}$ exchange are enhanced compared with those in Ref. 23. In particular, $K^{*}$ exchange becomes dominant in $\gamma p \rightarrow \bar{K}^{0} \Theta^{+}$, where the contact diagram and $t$-channel kaon exchange are absent. The cross sections also show strong dependence on the relative signs of the $K^{*}$ exchanges as illustrated by the dashed and dotted curves. Although simple $K$ and $K^{*}$ exchanges in this model are constructive, the generalisation to Regge trajectories leads to a non-trivial relative phase, though the relative strength of three is preserved due to its spin dependent origin. Hence the cross section should be considered as lying within the range covered by the dashed and dotted curves. This feature is consistent with other model calculations. If data continue to confirm the early suggestions of comparable rates for the $\Theta^{+}$production on the neutron [1] and proton target [4, 5], this would be consistent with a strong contribution from $K^{*}$ exchange.

In Fig. 3] total cross sections for $\Sigma_{5}(\overline{\mathbf{1 0}})$ in four reactions are presented. The production of $\Sigma_{5}(\overline{\mathbf{1 0}})$ is considered with a mass of $1.7 \mathrm{GeV}$. Cross sections for $\Sigma_{5}(\mathbf{8})$ production in general are relatively suppressed by a factor of two compared with those for $\Sigma_{5}(\overline{\mathbf{1 0}})$. The EM couplings for proton and neutron also result in cross section differences. This feature is shown by comparing Fig. B(a) to B(b), or comparing Fig. B(c) to B(d).

These figures show that photoproduction cross sections for $\Sigma_{5}(\overline{\mathbf{1 0}})$ and $\Sigma_{5}(\mathbf{8})$ are much smaller than for $\Theta^{+}$ production. The suppression comes from both the flavour coupling factors as shown in Table 【 and kinematic 
effects in these low energy experiments; e.g., the cross section difference between Fig. 2(b) and Fig. 31(d) arises mainly from the different pentaquark masses. At higher energies, such as at HERMES [7], only the flavour coupling suppression is expected. So the relative production rate for an ideal mixing $\Sigma_{d}$ between $\Sigma_{5}(\overline{\mathbf{1 0}})$ and $\Sigma_{5}(\mathbf{8})$, will lead to $\sigma\left(\gamma n \rightarrow K^{+} \Sigma_{d}^{-}\right) / \sigma\left(\gamma n \rightarrow K^{-} \Theta^{+}\right) \sim \sigma\left(\gamma p \rightarrow K^{0} \Sigma_{d}^{+}\right) / \sigma\left(\gamma p \rightarrow \bar{K}^{0} \Theta^{+}\right) \sim 1 / 2$.

The analogous cross sections for photoproduction of $\Lambda_{5}$ with a commonly assumed mass of $1.65 \mathrm{GeV}$ are shown in Fig. 4 The qualitative similarity and quantitative difference between $\gamma p \rightarrow K^{+} \Sigma_{5}^{0}$ [Fig. B(b)] and $\gamma p \rightarrow K^{+} \Lambda_{5}$ [Fig. [ (a)] is again due to the flavour coupling and kinematic effects. If the spin-orbit partner $\Lambda_{5}(J=3 / 2)$ is below $1.9 \mathrm{GeV}$, and the fall-apart dynamics is correct, then this could be another narrow unmixed pentaquark [16], whose photoproduction cross section should be similar or larger than those in Fig. 目

Figures 2 3] and 4have shown the cross sections as a function of energy including the Born terms and $K^{*}$ exchanges. As repeatedly stressed, these are strongly model-dependent far above threshold due to the lack of Reggeisation. However, visual inspection shows that the ratios of $\Sigma_{5}$ and $\Lambda_{5}$ to $\Theta^{+}$as a function of $E_{\gamma}$ is much less sensitive to the $K^{*}$ exchanges than the cross sections. They are thus instructive for our purpose of quantitizing the production rates of other pentaquark states based on the limited knowledge about the $\Theta^{+}$.

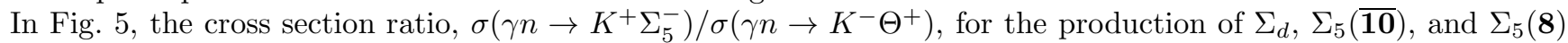
to the $\Theta^{+}$are presented. The kinematic effects are shown by the energy evolution of the ratios. If $\Sigma_{5}$ has the same mass as $\Theta^{+}$, the ratios would be constants $1 / 2,1 / 3$, and $1 / 6$ for $\Sigma_{d}, \Sigma_{5}(\overline{\mathbf{1 0}})$, and $\Sigma_{5}(\mathbf{8})$, respectively, as discussed earlier. Due to the mass difference, such constant ratios occur at much higher energies as shown by the convergence of the ratios for the Born terms and the charged $K^{*}$ exchanges.

The relative insensitivity of the production ratios of $\Sigma_{5}, \Lambda_{5}$ relative to the $\Theta^{+}$is also useful for estimating the pentaquark production rates taking into account the fact that their masses have not been determined yet. Although ideal mixing gives $\Sigma_{d}$ the same proportion of strange and nonstrange flavour masses such that naively one might expect $M_{\Sigma_{d}}=M_{\Theta}$, a potential mass difference can arise due to the $\bar{s}$ being isolated from the (qqqq) in the $\Theta^{+}$ whereas it is in a diquark in the $\Sigma_{d}$.

We show in Fig. G(a)-(c) the cross section ratios of $\Sigma_{d}, \Sigma_{5}(\overline{\mathbf{1 0}})$, and $\Sigma_{5}(\mathbf{8})$ to $\Theta^{+}$at $E_{\gamma}=3 \mathrm{GeV}$ (c.m. energy $W \simeq 2.55 \mathrm{GeV})$ as a function of their masses within a theoretically predicted range: $\Sigma_{5}(\overline{\mathbf{1 0}})$ and $\Sigma_{5}(\mathbf{8})$ are considered to have a mass range of 1.65 to $1.75 \mathrm{GeV}$; an ideally mixed $\Sigma_{d}$ is taken to be within 1.54 to $1.7 \mathrm{GeV}$ and $\Sigma_{s}$ in 1.8 to $2 \mathrm{GeV}$. Interestingly, it shows that in this energy region, the cross section rates are not sensitive to the pentaquark masses. Hence, one should be cautious with interpreting any narrow baryon resonance observed in $p K_{S}^{0}$ invariant mass in the absence of an accompanying strange hadron that may constrain the strangeness of the narrow baryon. For example, in the HERMES data in particular [7], we would expect the presence of $\Sigma^{+}$accompanying any $\Theta^{+}$.

The production of $\Sigma_{s}$ cannot be studied here since it is now decoupled to the $N K$ channel.

The $\Lambda_{5}$ production ratio $\left(\gamma p \rightarrow K^{+} \Lambda_{5}\right)$ to the $\Theta^{+}\left(\gamma n \rightarrow K^{-} \Theta^{+}\right)$with a varying mass range 1.54 to $1.7 \mathrm{GeV}$ is presented in Fig. [6 (d). It shows that the $K^{*}$ exchange could be the key process for the production of this state if any sizeable signals are observed in experiment.

These ideas can be generalised to the cases of $N_{5}$ production with $g_{N_{5} N \pi(\rho)}=g_{\Theta N K\left(K^{*}\right)}$. For the case of ideal mixing, the decomposition gives:

$$
\left\{\begin{array}{l}
p_{d} \rightarrow-\frac{1}{6}\left\{3 p \pi^{0}-3 \sqrt{2} n \pi^{+}+\sqrt{6} p \eta_{1}+\sqrt{3} p \eta_{8}\right\} \\
n_{u} \rightarrow \frac{1}{6}\left\{-3 \sqrt{2} p \pi^{-}+3 n \pi^{0}+\sqrt{6} n \eta_{1}+\sqrt{3} n \eta_{8}\right\}
\end{array},\right.
$$

for the proton and neutron pentaquarks. If we consider the non-strange pentaquark $p_{d}$ and $n_{u}$ with mass assumed at $1.44 \mathrm{GeV}$ [17], the cross sections for $\gamma p \rightarrow n_{u} \pi^{+}$and $\gamma n \rightarrow \pi^{-} p_{d}$ peak at around $70 \mathrm{nb}$ when $E_{\gamma} \sim 1.2 \mathrm{GeV}$. In Fig. [7] (a), the cross section for $\gamma n \rightarrow \pi^{-} p_{d}$ is shown. The magnitude of cross sections is larger than for $\Theta^{+} K^{-}$primarily because of the light $\pi$ mass. Also, the cross section for $\gamma p \rightarrow \pi^{+} n_{u}$ is slightly larger than for the neutron reaction due to the presence of the electric interaction. Here, again the energy dependence of the cross sections is strongly model-dependent due to the lack of Reggeisation; but the ratios are still robust. In Fig. [7 (b), we show the relative rates of $p_{d}$ to $\Theta^{+}$for the mass range 1.4 to $1.7 \mathrm{GeV}$ at $E_{\gamma}=3 \mathrm{GeV}(W \simeq 2.55 \mathrm{GeV})$.

In summary: The work of Ref. [16] provides a framework for systematically constructing pentaquark flavour wavefunctions with explicit constraints on their relative phases and coupling strengths in their strong decays into conventional octet baryons and mesons. This then leads to a quantitative estimate of other pentaquark photoproduction rates based on the presently limited experimental and theoretical information about the $\Theta^{+}$. In particular, continued observation of $\Theta^{+}$, and absence of $\Sigma_{5}, \Lambda_{5}$ at these rates would imply that the pentaquark dynamics and masses are outside the currently assumed range, or that these states do not exist. Also, any experimental tests of the selection rules should shed light on the pentaquark internal dynamics, in particular, the fall-apart mechanism, which has been suggested to be important for understanding the narrow widths.

Even if pentaquarks with non-exotic quantum numbers (such as $\Lambda_{5}, \Sigma_{5}$ and $N_{5}$ ) mix with conventional $q q q$ states, and as such are lost to a simple spectroscopic search, the photoproduction cross sections for these physical states should be at least as big as those calculated here, courtesy of any $B_{5}$ content. Thus, if photoproduction of states 
such as $\Lambda(1600)$ or $\Sigma(1660)$ is not seen at levels commensurate with Figs. 3] [5] and [6] and no manifest pentaquark analogues are seen either, then one would need to re-evaluate existing assumptions about pentaquark spectroscopy and dynamics.

This work is supported, in part, by grants from the U.K. Particle Physics and Astronomy Research Council, and the EU-TMR program "Eurodice", HPRN-CT-2002-00311, and the Engineering and Physical Sciences Research Council (Grant No. GR/R78633/01). FEC is indebted to A. Donnachie for discussions about Reggeised photoproduction.

[1] T. Nakano et al. [LEPS Collaboration], Phys. Rev. Lett. 91, 012002 (2003).

[2] V. Barmin et al. [DIANA Collaboration], Phys. Atom. Nucl. 66, 1715 (2003) [Yad. Fiz. 66, 1763 (2003)].

[3] S. Stepanyan et al. [CLAS Collaboration], Phys. Rev. Lett. 91, 252001 (2003).

[4] J. Barth et al. [SAPHIR Collaboration], Phys. Lett. B 572, 127 (2003).

[5] V. Kubarovsky et al. [CLAS Collaboration], Phys. Rev. Lett. 92, 032001 (2004) [Erratum-ibid. 92, 049902 (2004)].

[6] A.E. Asratyan, A.G. Dolgolenko, and M.A. Kubantsev, hep-ex/0309042

[7] A. Airapetian et al. [HERMES Collaboration], hep-ex/0312044

[8] A. Aleev et al. [SVD Collaboration], hep-ex/0401024

[9] M. Abdel-Bary et al. [COSY-TOF Collaboration], hep-ex/0403011

[10] A. R. Dzierba, D. Krop, M. Swat, S. Teige, and A. P. Szczepaniak, hep-ph/0311125 R. Cahn and G. Trilling, Phys. Rev. D 69, 011501 (2004); hep-ph/0311245

[11] J.Z. Bai et al. [BES Collaboration], hep-ex/0402012

[12] F.E. Close, Conference summary talk at Hadron03, hep-ph/0311087

[13] C. Alt et al., [NA49 Collaboration], Phys. Rev. Lett. 92, 042003 (2004); hep-ex/0310014

[14] H.G. Fischer and S. Wenig, hep-ex/0401014

[15] B.K. Jennings and K. Maltman, hep-ph/0308286 C.E. Carlson, C. D. Carone, H. J. Kwee and V. Nazaryan, hep-ph/0312325 F. Buccella and P. Sorba, hep-ph/0401083

[16] F.E. Close and J.J. Dudek, Phys. Lett. B (in press); hep-ph/0401192

[17] R. Jaffe and F. Wilczek, Phys. Rev. Lett. 91, 232003 (2003) hep-ph/0307341.

[18] M.V. Polyakov and A. Rathke, Eur. Phys. J. A 18, 691 (2003).

[19] T. Hyodo, A. Hosaka, and E. Oset, Phys. Lett. B 579, 290 (2004).

[20] S.I. Nam, A. Hosaka, and H.-Ch. Kim, Phys. Lett. B 579, 43 (2004); hep-ph/0403009

[21] W. Liu and C.M. Ko, Phys. Rev. C 68, 045203 (2003); nucl-ph/0309023

[22] Y. Oh, H. Kim, and S.H. Lee, Phys. Rev. D 69, 014009 (2004); hep-ph/0312229

[23] Q. Zhao, Phys. Rev. D (in press); hep-ph/0310350

[24] Q. Zhao and J.S. Al-Khalili, Phys. Lett. B 585, 91 (2004) hep-ph/0312348.

[25] K. Nakayama and K. Tsushima, Phys. Lett. B 583, 269 (2004).

[26] B.-G. Yu, T.-K. Choi, and C.-R. Ji, nucl-th/0312075

[27] S.-H. Lee, H. Kim, and Y. Oh, hep-ph/0402135

[28] D. E. Groom et al. (Particle Data Group), Eur. Phys. J. C 15, 1 (2000).

[29] P.-Z. Huang, W.-Z. Deng, X.-L. Chen and S.-L. Zhu, hep-ph/0311108

[30] Y.-R. Liu, P.-Z. Huang, W.-Z. Deng, X.-L. Chen, and S.-L. Zhu, hep-ph/0312074

[31] J.J.Dudek and F.E.Close, Phys. Lett. B 583, 278 (2004).

[32] H.-C. Kim and M. Praszalowicz, Phys. Lett. B 585, 99 (2004).

[33] R. Bijker, M.M. Giannini, and E. Santopinto, hep-ph/0403029 


\begin{tabular}{c|c|c}
\hline Pentaquark & Representation & Decomposition into octet baryons and mesons \\
\hline$\Theta^{+}$ & $\overline{\mathbf{1 0}}$ & $\frac{1}{\sqrt{2}}\left\{p K^{0}-n K^{+}\right\}$ \\
\hline$\Sigma_{5}^{+}$ & $\overline{\mathbf{1 0}}$ & $\frac{1}{2 \sqrt{3}}\left\{\sqrt{3} \Sigma^{+} \eta_{8}+\Sigma^{+} \pi^{0}-\Sigma^{0} \pi^{+}-\sqrt{2} p \bar{K}^{0}+\sqrt{3} \Lambda \pi^{+}+\sqrt{2} \Xi^{0} K^{+}\right\}$ \\
& $\mathbf{8}_{5}$ & $\frac{1}{2 \sqrt{6}}\left\{\sqrt{6} \Sigma^{+} \eta_{1}-\sqrt{3} \Sigma^{+} \eta_{8}+\Sigma^{+} \pi^{0}-\Sigma^{0} \pi^{+}-\sqrt{2} p \bar{K}^{0}+\sqrt{3} \Lambda \pi^{+}+2 \sqrt{2} \Xi^{0} K^{+}\right\}$ \\
\hline$\Sigma_{5}^{0}$ & $\overline{\mathbf{1 0}}$ & $-\frac{1}{2 \sqrt{3}}\left\{\sqrt{3} \Sigma^{0} \eta_{8}+n \bar{K}^{0}+p K^{-}-\sqrt{3} \Lambda \pi^{0}+\Sigma^{-} \pi^{+}-\Sigma^{+} \pi^{-}-\Xi^{-} K^{+}-\Xi^{0} K^{0}\right\}$ \\
& $\mathbf{8}_{5}$ & $-\frac{1}{2 \sqrt{6}}\left\{-\sqrt{3} \Sigma^{0} \eta_{8}+\sqrt{6} \Sigma^{0} \eta_{1}+n \bar{K}^{0}+p K^{-}-\sqrt{3} \Lambda \pi^{0}+\Sigma^{-} \pi^{+}-\Sigma^{+} \pi^{-}+2 \Xi^{-} K^{+}+2 \Xi^{0} K^{0}\right\}$ \\
\hline$\Lambda_{5}$ & $\mathbf{8}_{5}$ & $\frac{1}{2 \sqrt{2}}\left\{\Lambda \eta_{8}+\sqrt{2} \Lambda \eta_{1}+n \bar{K}^{0}-p K^{-}+\Sigma^{-} \pi^{+}+\Sigma^{+} \pi^{-}-\Sigma^{0} \pi^{0}\right\}$ \\
\hline$p_{5}$ & $\overline{\mathbf{1 0}}$ & $-\frac{1}{2 \sqrt{3}}\left\{\sqrt{3} p \eta_{8}-\sqrt{2} n \pi^{+}+p \pi^{0}+\sqrt{3} \Lambda K^{+}+\sqrt{2} \Sigma^{+} K^{0}-\Sigma^{0} K^{+}\right\}$ \\
& $\mathbf{8}_{5}$ & $-\frac{1}{2 \sqrt{6}}\left\{-\sqrt{6} p \eta_{1}+2 \sqrt{2} n \pi^{+}-2 p \pi^{0}+\sqrt{3} \Lambda K^{+}+\sqrt{2} \Sigma^{+} K^{0}-\Sigma^{0} K^{+}\right\}$ \\
\hline$n_{5}$ & $\overline{\mathbf{1 0}}$ & $\frac{1}{2 \sqrt{3}}\left\{\sqrt{3} n \eta_{8}+n \pi^{0}-\sqrt{2} p \pi^{-}-\sqrt{3} \Lambda K^{0}+\sqrt{2} \Sigma^{-} K^{+}-\Sigma^{0} K^{0}\right\}$ \\
& $\mathbf{8}_{5}$ & $\frac{1}{2 \sqrt{6}}\left\{-\sqrt{6} n \eta_{1}-2 n \pi^{0}+2 \sqrt{2} p \pi^{-}-\sqrt{3} \Lambda K^{0}+\sqrt{2} \Sigma^{-} K^{+}-\Sigma^{0} K^{0}\right\}$ \\
\hline
\end{tabular}

TABLE I: Decomposition of pentaquark flavour wavefunctions into octet baryons and mesons.

\begin{tabular}{c|c|c|c}
\hline Decay channels & $\Theta^{+}$ & $\Sigma^{+}(\overline{\mathbf{1 0}})$ & $\Sigma^{+}\left(\mathbf{8}_{5}\right)$ \\
\hline$p K^{0}$ & 0.5 & $*$ & $*$ \\
\hline$n K^{+}$ & 0.5 & $*$ & $*$ \\
\hline$p \bar{K}^{0}$ & $*$ & 0.57 & 0.28 \\
\hline$\Sigma^{+} \pi^{0}$ & $*$ & 0.47 & 0.23 \\
\hline$\Sigma^{0} \pi^{+}$ & $*$ & 0.47 & 0.23 \\
\hline$\Sigma^{+} \eta_{8}$ & $*$ & m.b.t. & m.b.t. \\
\hline$\Lambda \pi^{+}$ & $*$ & 1.74 & 0.87 \\
\hline$\Xi^{0} K^{+}$ & $*$ & m.b.t & m.b.t. \\
\hline$\Sigma^{+} \eta_{1}$ & $*$ & $*$ & m.b.t \\
\hline Total $(\mathrm{MeV})$ & 1.0 & 3.2 & 1.6 \\
\hline \hline
\end{tabular}

TABLE II: Partial and isospin averaged total widths for $\Theta^{+}$and $\Sigma_{5}^{+}$. Symbol “*” stands for the decoupled channels, while abbreviation "m.b.t" for the case that the mass of pentaquark is below the threshold of a decay channel. Total masses $M_{\Lambda_{5}}=1.65 \mathrm{GeV}$ and $M_{\Sigma_{5}}=1.7 \mathrm{GeV}$ are adopted. We do not consider $\Sigma^{*} \pi$ as this mode is forbidden by selection rules [16]. For the purpose of this table we identify $\eta_{8}$ and $\eta_{1}$ with $\eta$ and $\eta^{\prime}$, respectively.

\begin{tabular}{c|c|c|c}
\hline Decay channels & $\Lambda\left(\mathbf{8}_{5}\right)$ & $\Sigma^{0}(\overline{\mathbf{1 0}})$ & $\Sigma^{0}\left(\mathbf{8}_{5}\right)$ \\
\hline$n \bar{K}^{0}$ & 0.32 & 0.28 & 0.14 \\
\hline$p K^{-}$ & 0.32 & 0.28 & 0.14 \\
\hline$\Lambda \eta_{1}$ & m.b.t & $*$ & $*$ \\
\hline$\Lambda \eta_{8}$ & m.b.t & $*$ & $*$ \\
\hline$\Lambda \pi^{0}$ & $*$ & 1.75 & 0.87 \\
\hline$\Sigma^{0} \pi^{0}$ & 0.54 & $*$ & $*$ \\
\hline$\Sigma^{-} \pi^{+}$ & 0.54 & 0.46 & 0.23 \\
\hline$\Sigma^{+} \pi^{-}$ & 0.54 & 0.46 & 0.23 \\
\hline$\Sigma^{0} \eta_{1}$ & $*$ & $*$ & m.b.t \\
\hline$\Sigma^{0} \eta_{8}$ & $*$ & m.b.t. & m.b.t. \\
\hline$\Xi^{0} K^{0}$ & $*$ & m.b.t & m.b.t \\
\hline$\Xi^{-} K^{+}$ & $*$ & m.b.t & m.b.t \\
\hline Total $(\mathrm{MeV})$ & 2.3 & 3.2 & 1.6 \\
\hline \hline
\end{tabular}

TABLE III: Partial and isospin averaged total widths for $\Lambda_{5}$ and $\Sigma_{5}^{0}$. Notations are the same as Table II The equality of the widths for $\Sigma_{5}^{0}$ and $\Sigma_{5}^{+}$is a numerical coincidence at these kinematics. 


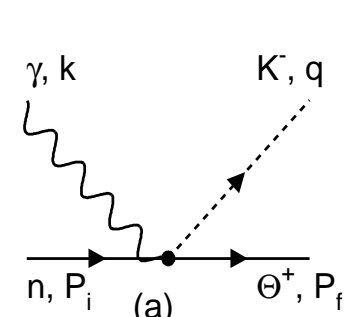

(a)

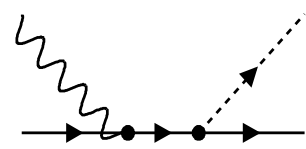

(c)

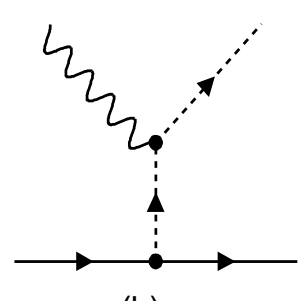

(b)

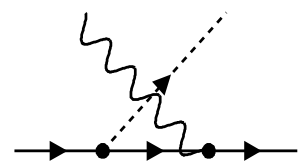

(d)

FIG. 1: Feynman diagrams for pentaquark photoproduction in the Born approximation. (a) and (b) will only contribute to the processes with charged meson produced.
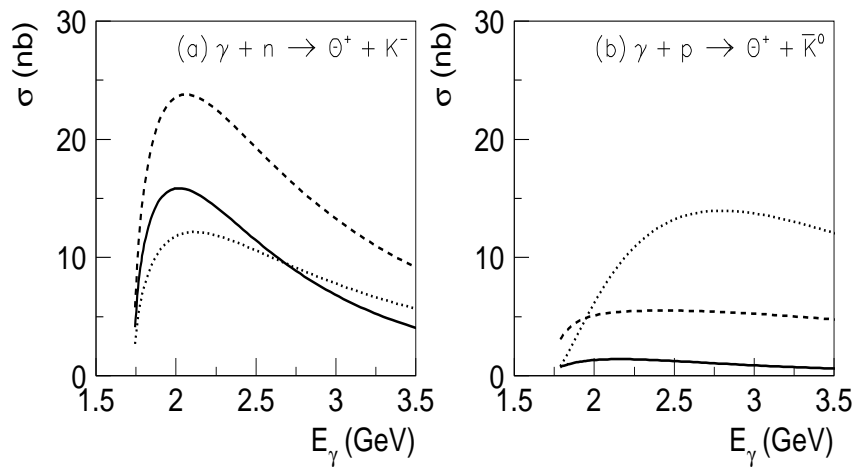

FIG. 2: Total cross sections for $\Theta^{+}$photoproduction in $\gamma n \rightarrow \Theta^{+} K^{-}$and $\gamma p \rightarrow \Theta^{+} \bar{K}^{0}$ with the total width of 1 MeV. The solid curves are results in the Born limit without $K^{*}$ exchanges, while the dashed and dotted curves are results including $K^{*}$ exchanges of different signs. For other total widths $\Gamma_{T}$ (in $\mathrm{MeV}$ ), the cross sections can be rescaled by $\Gamma_{T} /(1 \mathrm{MeV})$. Thus, e.g., if $\Gamma_{T}=10 \mathrm{MeV}$, the $\sigma$ will be 10 times larger. 

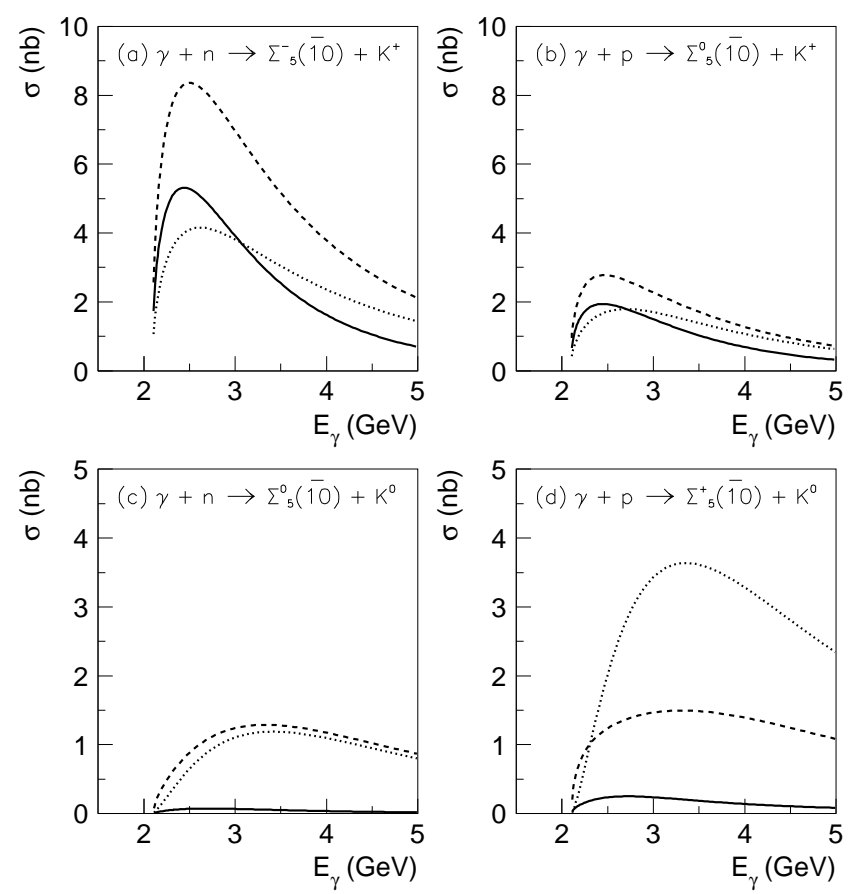

FIG. 3: Total cross sections for $\Sigma_{5}(\overline{\mathbf{1 0}})$ photoproduction in four reactions. Notations are the same as Fig. 2
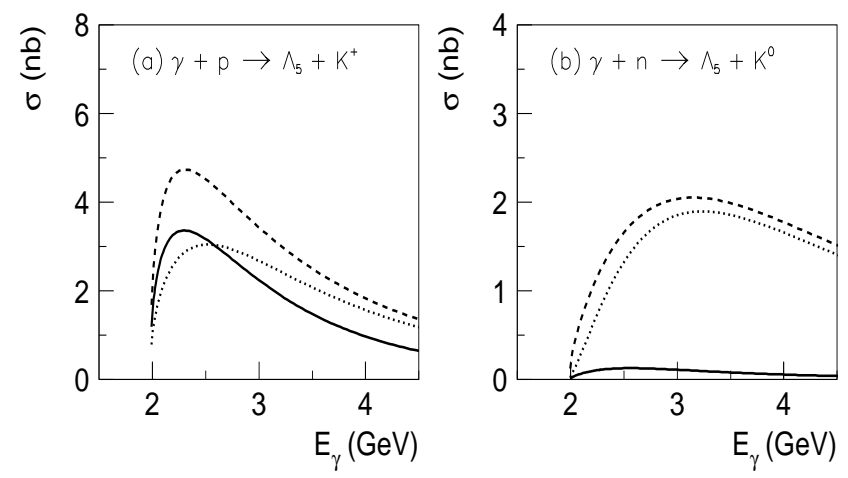

FIG. 4: Total cross sections for $\Lambda_{5}$ photoproduction on the proton and neutron. Notations are the same as Fig. 2 


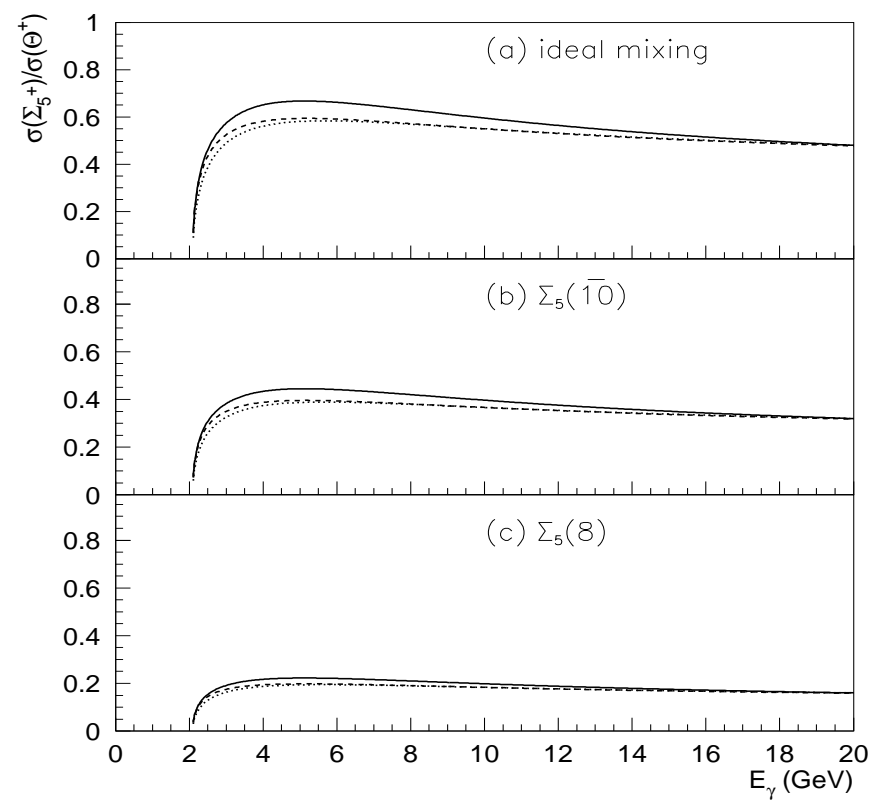

FIG. 5: Energy dependence of the cross section ratios for the ideally mixed $\Sigma_{d}^{+}, \Sigma_{5}^{+}(\overline{\mathbf{1 0}})$, and $\Sigma_{5}^{+}(\mathbf{8})$. The Born terms are shown by the solid curves, while the dashed and dotted curves denote the inclusion of $K^{*}$ exchange with \pm signs.
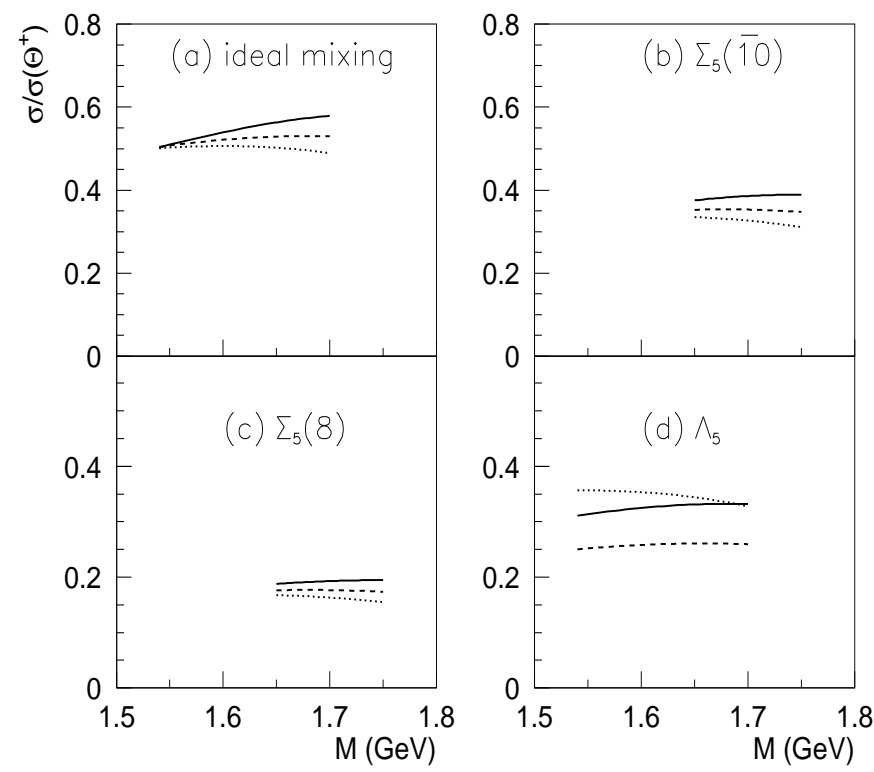

FIG. 6: The cross section ratios for the ideally mixed $\Sigma_{d}^{+}, \Sigma_{5}^{+}(\overline{\mathbf{1 0}}), \Sigma_{5}^{+}(\mathbf{8})$, and $\Lambda_{5}$ as a function of the varying pentaquark masses at $E_{\gamma}=3 \mathrm{GeV}$. The Born terms are shown by the solid curves, while the dashed and dotted curves denote the inclusion of $K^{*}$ exchange with \pm signs. 

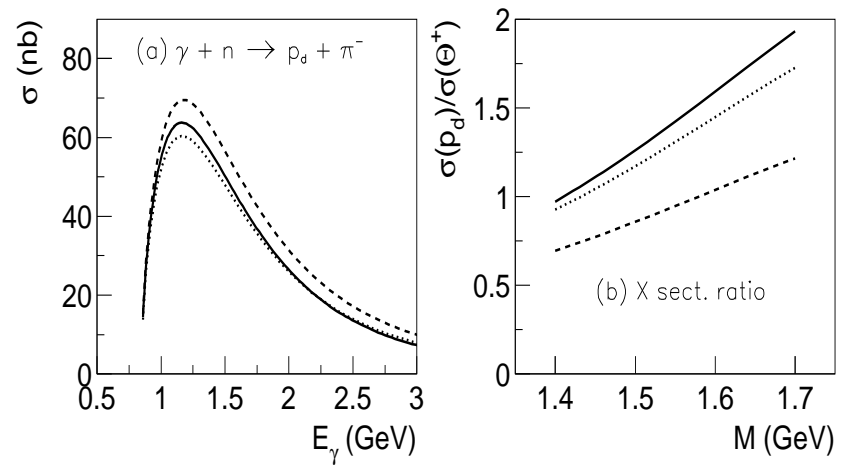

FIG. 7: (a) Total cross sections for the nucleon pentaquark $p_{d}$ photoproduction with ideal mixing. (b) The cross section ratios to the $\Theta^{+}$as a function of the varying $p_{d}$ mass at $E_{\gamma}=3 \mathrm{GeV}$. The solid curves are results in the Born limit without pion exchanges, while the dashed and dotted curves are results including $\rho$ exchanges of different signs. 